\title{
A NOTE ON PERIODIC SOLUTIONS OF SECOND ORDER DIFFERENTIAL EQUATIONS WITHOUT DAMPING
}

\section{GEORGE SEIFERT}

We consider the differential equation

$$
\ddot{x}+g(x)=p(t),
$$

where, in addition to satisfying conditions which insure existence and uniqueness of solutions, $g(x)$ and $p(t)$ satisfy the following conditions:

(i) there exist positive constants $\tau$ and $k$ such that for all $t, p(t)$ $=p(t+\tau)$ and $|p(t)|<k$;

(ii) $g(x)$ is continuous, increasing, $g(0)=0$, and for $|x|$ sufficiently large, $|g(x)|>k$.

We show that for $\tau$ sufficiently small, Equation (1) has a solution $x(t)$ of period $\tau$. Our method of proof consists of showing that a certain mapping of the phase plane of Equation (1) has a fixed point, not by use of the Brouwer theorem, but by a somewhat more general result concerning the index of the bounding curve of a simply-connected region relative to a vector field induced by the mapping; $c f$. [1, p. 337].

We consider Equation (1) in terms of the system:

$$
\dot{x}=y, \quad \dot{y}=p(t)-g(x),
$$

the solutions $(x(t), y(t))$ of which define the so-called phase trajectories of the system. Let $x=a$ be the root of $g(x)=-k$, and $x=b$ be the root of $g(x)=k$. That these roots exist and are unique follows immediately from (ii). We define

$$
G(x)=\int_{0}^{x} g(v) d v
$$

and using (ii), we observe that the graph of $y=G(x)$ has the following properties:

(iii) it is tangent to the $x$-axis at the origin and is everywhere concave up;

(iv) its slope $G^{\prime}(x)=g(x)$ is such that $-k<G^{\prime}(x)<k$ for $a<x<b$, $G^{\prime}(x)>k$ for $x>b$, and $G^{\prime}(x)<-k$ for $x<a$.

Hence, there clearly exists a positive constant $C_{1}$ such that the line $y=k x+C_{1}$ intersects the graph of $y=G(x)$ at exactly two points whose abscissas $r_{2}$ and $r_{3}$ are such that $r_{2}<a<b<r_{3}$. Similarly there exists a positive constant $C_{2}$ such that the line $y=-k x+C_{2}$ intersects the graph of $y=G(x)$ at exactly two points whose abscissas $r_{1}$ and $r_{4}$ are such that $r_{1}<r_{2}<r_{3}<r_{4}$.

Received by the editors June 2, 1958 and, in revised form, September 8, 1958. 
We denote by $\Gamma_{1}$ the open arc defined by $y^{2} / 2+G(x)=k x+C_{1}$, $y>0$, by $\Gamma_{2}$ the open arc defined by $y^{2} / 2+G(x)=-k x+C_{2}, y<0$, and observe that the end points of these arcs are at $\left(r_{2}, 0\right),\left(r_{3}, 0\right)$ and $\left(r_{1}, 0\right),\left(r_{4}, 0\right)$ respectively.

Lemma 1. The slope of any phase trajectory of (2) at a point of either $\Gamma_{1}$ or $\Gamma_{2}$ is less than the slope of the curve $\Gamma_{1}$ or $\Gamma_{2}$ at that point.

Proof. We prove the lemma only for a point $\left(x_{0}, y_{0}\right)$ on $\Gamma_{1}$; the proof for a point on $\Gamma_{2}$ is entirely similar, and is omitted. Clearly the slope of $\Gamma_{1}$ at $\left(x_{0}, y_{0}\right)$ is given by $\left(k-g\left(x_{0}\right)\right) / y_{0}$, while the slope of the trajectory of (2) at $\left(x_{0}, y_{0}\right)$ for $t=t_{0}$ is given by $\left(p\left(t_{0}\right)-g\left(x_{0}\right)\right) / y_{0}$, which is clearly less than the slope of $\Gamma_{1}$ there. This proves the lemma.

We now denote by $P_{1}, P_{2}$, and $P_{3}$ the points $\left(r_{1}, 0\right)$ and $\left(r_{3}, 0\right)$ respectively; by $Q$ the intersection of the line $x=r_{3}$ with $\Gamma_{2}$, and by $R$ the closed region bounded by the curves $\Gamma_{1}, \Gamma_{2}$ from $P_{1}$ to $Q$, and the line segments $P_{1} P_{2}, P_{3} Q$. We now prove another simple

Lemma 2. Let $\left(x_{0}, y_{0}\right)$ be a point on the boundary of $R$, and let $(x(t), y(t))$ be the solution of (2) for which $x(0)=x_{0}, y(0)=y_{0}$. Then the trajectory of this solution can only leave $R$ for some $t_{0} \geqq 0$, if $r_{1} \leqq x\left(t_{0}\right)$ $\leqq r_{2}$, and $y\left(t_{0}\right)=0$.

Proof. Due to Lemma 1 , we need only prove that if $\left(x_{0}, y_{0}\right)$ is on the segment $P_{3} Q$, then the trajectory at $\left(x_{0}, y_{0}\right)$ for some arbitrary $t=t_{0}>0$ passes first into $R$ as $t$ increases from $t_{0}$. This is obvious if $\left(x_{0}, y_{0}\right)$ is $Q$ since $Q$ is on $\Gamma_{2}$; it is also obvious for $\left(x_{0}, y_{0}\right)$ between $P_{3}$ and $Q$ since then $y_{0}<0$, and hence $\dot{x}\left(t_{0}\right)=y\left(t_{0}\right)<0$. Finally if $\left(x_{0}, y_{0}\right)$ is $P_{3}$, then since $y\left(t_{0}\right)=-g\left(x\left(t_{0}\right)\right)+p\left(t_{0}\right)<0, y(t)<0$ for $t>t_{0}, t-t_{0}$ sufficiently small; hence $x(t)<x\left(t_{0}\right)$ also for $t>t_{0}, t-t_{0}$ sufficiently small.

This essentially proves the lemma.

We now define $M=\left|2\left(G(a)+k a-C_{2}\right)\right|^{1 / 2}, m=\left(2\left(G(a)-k a-C_{1}\right)\right)^{1 / 2}$, $d=a-r_{2}$, and $k=\max _{r_{1} \leq x \leq 0}(-g(x)+k)$ and prove the following:

THEOREM. In terms of the definitions above, if $\tau<\min (d / M, m / K)$, then system (2), hence Equation (1), has a solution of period $\tau$.

Proof. We define the mapping $T$ of the $(x, y)$ plane into itself as follows: $T\left(x_{0}, y_{0}\right)=\left(x_{1}, y_{1}\right)$ where $x_{1}=x(\tau), y_{1}=y(\tau)$, and $(x(t), y(t))$ is the solution of (2) such that $x(0)=0, y(0)=0$. We show that this mapping has a fixed point; this will prove the theorem; cf. [1, p. 270].

We consider the vector field defined by this mapping, and will show that the index of the boundary of $R$ relative of this field (cf. $[1$, p. 337]) is +1 ; this will prove that $R$ contains a critical point of this field; i.e., that the mapping $T$ has a fixed point in $R$. 
Let $\left(x_{0}, y_{0}\right)$ be a point of $\Gamma_{1}$; we show first that $T\left(x_{0}, y_{0}\right)$ is in $R$. For if not, there exists by Lemma 2 a smallest $t_{1}, 0<t_{1}<\tau$, such that $y\left(t_{1}\right)=0, r_{1} \leqq x\left(t_{1}\right) \leqq r_{2}$; here as throughout this proof $(x(t), y(t))$ is the solution of (2) used in the definition of $T$. Suppose first $x_{0} \geqq a$; then clearly for $0<t<t_{1},|y(t)|<M$. Since $\dot{x}(t)=y(t)$,

$$
\left|x\left(t_{1}\right)-x_{0}\right|=\left|\int_{0}^{t_{1}} y(t) d t\right|<M \tau .
$$

However, $\left|x\left(t_{1}\right)-x_{0}\right| \geqq a-r_{2}=d$; i.e., $d<\tau M$, a contradiction. If $r_{2}<x_{0}<a$, then since $\dot{y}=-g(x)+p(t)>0$ for $x=x(t)<a$, there exists a $t_{0}>0$ such that $(x(t), y(t))$ is in $R$ for $0 \leqq t \leqq t_{0}$, and $x\left(t_{0}\right)=a$. From here, the argument toward a contradiction proceeds as above, and we omit it. In fact, if $\left(x_{0}, y_{0}\right)$ is on either the segment $P_{3} Q$ or the curve $\Gamma_{2}$ from $(a,-M)$ to $Q$, the same argument, in essence, applies to show that $T\left(x_{0}, y_{0}\right)$ is in $R$.

If $\left(x_{0}, y_{0}\right)$ is on $\Gamma_{2}$ while $x<a$, or on the segment $P_{1} P_{2}$, then if $T\left(x_{0}, y_{0}\right)=\left(x_{1}, y_{1}\right)$ is not in $R$, we show finally that $r_{1} \leqq x_{1} \leqq a, 0 \leqq y_{1} \leqq m$. In any case, there exists $t_{0}, 0 \leqq t_{0}<\tau$, such that $r_{1} \leqq x\left(t_{0}\right) \leqq r_{2}, y\left(t_{0}\right)=0$. If $x(t)<a$ for $t_{0}<t<\tau$, then clearly $x(t)>r_{1}$ for these values of $t$. Assume that for $t_{1}, t_{0}<t_{1}<\tau$, we have $y\left(t_{1}\right)>m$. Then since $\dot{y}=-g(x)$ $+p(t)$, we have

$$
y\left(t_{1}\right)-y\left(t_{0}\right)=\int_{t_{0}}^{t_{1}}(-g(x(t))+p(t)) d t<K \tau .
$$

But $y\left(t_{1}\right)-y\left(t_{0}\right)=y\left(t_{1}\right)>m$; hence $m<K \tau$, a contradiction, and we conclude that $x\left(t_{2}\right)=a$ for some $t_{2}, t_{0}<t_{2}<\tau$, while $0<y\left(t_{2}\right)<m$. Since $M>m$, and $x\left(t_{2}\right)-x\left(t_{0}\right)>d$, we easily arrive at a contradiction as before by integrating $\dot{x}(t)=y(t)$ from $t_{0}$ to $t_{2}$; we omit the details.

If the vector field $(x, y) \rightarrow T(x, y)$ has a critical point on the boundary of $R$, then by definition, the mapping has a fixed point there and there is nothing more to prove. We therefore assume that as $(x, y)$ moves in a counter-clockwise circuit around the boundary of $R$, the vector at each point of the boundary is nonzero; since it is also a continuous function of $(x, y)$ and in view of the location of the terminal point $T(x, y)$ for $(x, y)$ on the boundary of $R$ as established in the proof of this theorem, it follows that the index of this circuit relative to this field is +1 . This completes the proof of the theorem.

\section{REFERENCE}

1. S. Lefschetz, Differential equations: Goometric theory, Interscience Publishers, Inc., New York, 1957.

Iowa State College of Agriculture and Mechanic Arts 